
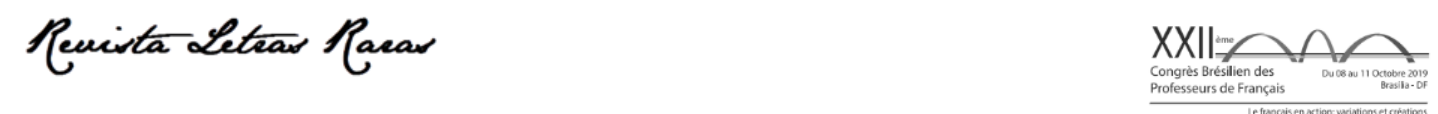

Actes du XXIlème Congrès brésilien des professeurs de français (dans Édition spéciale de la Revue Letras Raras 2020)

ISSN : 2317-2347 - v. 9, Dossier Spécial (2020)

Todo o conteúdo da RLR está licenciado sob Creative Commons Atribuição 4.0 Internacional

\title{
La littératie critique dans le cours de français : \\ la problématisation des identités sociales de genre et
}

\section{race/ethnie}

\section{Walesca Afonso Alves Pôrto*}

Master Recherche en Linguistique Apliquée (2016), Licence en Lettres Françaises (2009) et Portugaises (2013) à l'Université de Brasilia (UnB). Enseignante de FLE au Secrétariat d'État d'Éducation du District Fédéral (SEEDF).

iD https://orcid.org/0000-0003-0843-7648

Reçu le 26 oct. 2020. Approuvé le 06 nov. 2020.

\section{Comment citer cet article :}

AFONSO ALVES PÔRTO, Walesca. La littératie critique dans le cours de français: la problématisation des identités sociales de genre et race/ethnie. In: CONGRES BRESILIEN DES PROFESSEURS DE FRANÇAIS, 22., 2019, Brasília. Actes du XXIIème Congrès Brésilien des Professeurs de Français. Édition spéciale de la Revue Letras Raras: Campina Grande. EDUFCG. nov. 2020, p. 307-322.

\section{RÉSUMÉ}

Cet article a pour objectif d'analyser de quelle manière la littératie critique donne des opportunités pour la (re)construction des identités sociales de genre et race/ethnie tout en visant la formation citoyenne dans le cours de Français Langue Étrangère (FLE). L'objectif est de remettre en question les perceptions des participants par rapport à ces identités, en analysant les possibilités et les défis de cette perspective pédagogique. L'étude s'est déroulée en 2016 à l'Alliance Française de Brasilia et s'est basée sur les thèmes suivants : les identités comme construction (HALL, 2014) ; la littératie critique (FREIRE, 2011) ; et la recherche-action (BARBIER, 2007). La littératie critique a favorisé la remise en question des identités grâce aux activités développées. Le grand défi a surtout été la problématisation en soi lors des interactions. Les participants ont souligné l'importance de cette pratique pédagogique non seulement pour l'apprentissage de la langue française mais aussi pour la formation citoyenne.

MOTS-CLÉS : Enseignement de langues ; Identités sociales ; Littératie critique.

\section{Introduction}

L'école est un espace capital dans la construction de la vie sociale. En effet, c'est dans ce contexte, loin de la famille, où on entre en contact avec d'autres possibilités d'être (MOITA LOPES, 2012). Selon l'auteur, les cours de langues au Brésil sont encore loin de la vie sociale

Walescaporto@gmail.com 


\section{Revista Letear Racar}

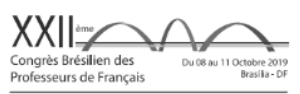

Actes du XXIlème Congrès brésilien des professeurs de français (dans Édition spéciale de la Revue Letras Raras 2020) ISSN : 2317-2347 - v. 9, Dossier Spécial (2020)

Todo o conteúdo da RLR está licenciado sob Creative Commons Atribuição 4.0 Internacional

et de l'engagement des apprenants dans le discours. D'où l'importance de travailler avec des thèmes qui touchent les apprenants eux-mêmes.

Étant donné que les discussions sur le genre sont toujours actuelles et que le racisme suscite également des inquiétudes en laissant une importante partie de la population à l'écart de la société, il devient essentiel de promouvoir des activités de formation et sensibilisation des apprenants à la citoyenneté démocratique dans le domaine de l'éducation.

De cette façon, on cherche à mettre en question les identités sociales de genre et race/ethnie dans la perspective de la littératie critique qui a pour finalité débattre des thèmes sociaux en classe tout en visant une formation citoyenne (MENEZES DE SOUZA, 2011 ; MONTE MÓR, 2015).

Les questions suivantes ont orienté cette recherche:

- Quelles sont les perceptions des participants sur les identités sociales de genre et race/ethnie?

- De quelle manière la littératie critique favorise des interactions encourageant la problématisation et la (re)construction des identités dans le cours de FLE ? Quelles en sont les possibilités et les défis?

- Quels sont les possibilités de cette pratique pédagogique dans la formation de citoyens critiques?

Dans les sections suivantes, nous aborderons les théories qui ont soutenu ce travail.

\section{Vers une perspective critique du langage}

La globalisation a changé notre façon de percevoir le monde. Grâce à l'internet, les informations vont d'un côté à l'autre presque spontanément, ce qui apporte une nouvelle configuration dans la vie contemporaine.

Dans ce contexte, nous sommes des sujets de multiples identités (HALL, 2014) qui correspondent aux plusieurs rôles que nous avons dans la société (WOODWORD, 2014). Ces identités sont construites « à travers le temps et l'espace » (NORTON, 2000, p. 5)․․ Tout cela se montre substantiel dans l'enseignement des langues une fois qu'en tant que pédagogues nous pouvons réfléchir sur les discours identitaires en classe. Étant donné que les identités ne

\footnotetext{
1 Traduction de l'auteure de : « across time and space ».
} 


\section{Revista Letear Racar}

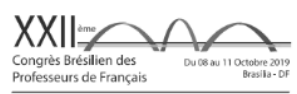

Actes du XXIlème Congrès brésilien des professeurs de français (dans Édition spéciale de la Revue Letras Raras 2020)

ISSN : 2317-2347 - v. 9, Dossier Spécial (2020)

Todo o conteúdo da RLR está licenciado sob Creative Commons Atribuição 4.0 Internacional

sont pas figées, il est possible de les (re)construire et mener les apprenants à s'engager à d'autres discours libres de préjugés (SILVA, 2014).

Dans cette étude, la langue est vue comme un moyen de représentation, ou alors comme pratique sociale, c'est-à-dire, comme une manière d'agir dans le monde et surtout sur les autres (FAIRCLOUGH, 2008). Le discours est construit socialement, il a toujours des idéologies (THOMPSON, 1990).

Ainsi, un enseignement critique a pour but lier des aspects de l'usage de la langue à des thèmes sociaux, politiques et culturels, tout en cherchant à comprendre comment les relations sociales ont été construites historiquement (PENNYCOOK, 2001). Nous partons aussi de la compréhension de l'éducation comme pratique de la liberté qui favorise la prise de conscience de la réalité (FREIRE, 2011). De même, nous nous penchons vers une pédagogie de la transgression (HOOKS, 2013) qui dépasse les frontières.

\section{La littératie critique}

La littératie est une pratique sociale qui demande un savoir être pour agir dans le monde. Selon Soares (1998), la littératie est le résultat du savoir lire et écrire. Elle concerne l'action dans les différents contextes sociaux. Les études sur la littératie critique au Brésil et ses avantages dans l'éducation originent des travaux du pédagogue Paulo Freire (2011) qui a proposé une éducation libre et critique à partir de la réalité des apprenants.

Menezes de Souza (2011) évoque que la littératie critique (LC) permet d'atteindre l'objectif pédagogique de préparer les apprenants à faire face à toute sorte de différence. Cette pratique propose débattre des questions sociales en classe afin de promouvoir le sens critique de l'apprenant pour qu'il puisse agir en tant que citoyen dans la société. Elle dialogue avec l'enseignement interculturel des langues dans lequel il est possible de dire que

[...] en s'attachant à la " dimension interculturelle » de l'enseignement des langues, on vise à faire des apprenants des locuteurs ou des médiateurs interculturels, capables de s'engager dans un cadre complexe et un contexte d'identités multiples, et d'éviter les stéréotypes accompagnant généralement la perception de l'autre dans une seule et unique identité (BYRAM ; GRIBKOVA ; STARKEY, 2002, p. 9-10). 

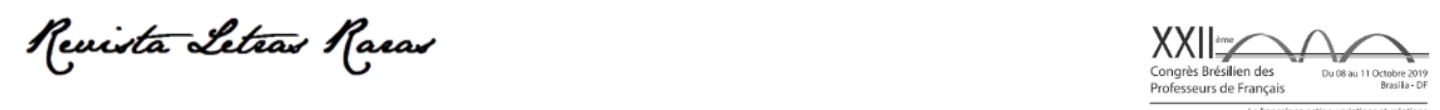

Actes du XXIlème Congrès brésilien des professeurs de français (dans Édition spéciale de la Revue Letras Raras 2020) ISSN : 2317-2347 - v. 9, Dossier Spécial (2020)

Todo o conteúdo da RLR está licenciado sob Creative Commons Atribuição 4.0 Internacional

De même, cette pratique dialogue avec la perspective actionnelle qui fait le lien entre la société et la classe : visant à former les apprenants aux valeurs citoyennes et aux compétences d'action sociale, l'enseignant leur demande d'agir en classe en tant qu'élèves comme des citoyens. (PUREN, 2018).

La LC n'est pas une méthodologie mais d'une façon d'agir en classe. Duboc (2015) propose de la travailler à partir des manuels, des outils pédagogiques déjà présents dans ce contexte éducationnel et de développer la réflexion des apprenants en problématisant les images et les textes y présents, allant au-delà de ce qui est proposé par le manuel, et facilitant l'engagement des apprenants dans le discours.

\section{Les identités sociales de genre et race/ethnie}

Dans cette recherche, les thèmes développés en classe sont le genre et la race/ethnie dont les identités sont socialement construites. Malgré les instruments juridiques créés pour interdire et sanctionner toute expression de violence domestique, comme la Loi Maria da Penha en 2006 ou encore les crimes de racisme, comme la Loi Caó en 1989, le Brésil reste l'un des pays les plus racistes et violents contre les femmes. C'est pour cela, qu'il se fait de plus en plus nécessaire de débattre ces questions à l'école.

D'abord il faut bien distinguer le genre et le sexe. Celui-ci est une donnée biologique, il est défini par les caractéristiques physiques et génétiques d'une personne, tandis que le genre est une construction sociale et décrit ce que la société, à une époque donnée, définit comme féminin et masculin. D'où la célèbre phrase de Simone de Beauvoir dans son œuvre : Le deuxième sexe écrit en 1949 « on ne naît pas femme, on le devient».

Le genre est donc une catégorie sociale instituée sur un corps et à partir de cela on définit les rôles des hommes et des femmes dans la société (SCOTT, 1995). Filles et garçons, par exemple, ne reçoivent pas la même éducation. Dès très tôt, la société définit ces rôles en attribuant aux filles et garçons des qualités et des comportements différents. Les adjectifs utilisés pour les caractériser sont un exemple de cette construction. Tout cela crée des stéréotypes qui vont forcément les influencer dans la vie adulte. Ces études nous aident à mieux comprendre comment les inégalités sont construites et renforcées chaque jour. L'école se montre donc un espace important pour débattre le thème et un moyen de proposer aux apprenants une éducation plus égalitaire. 

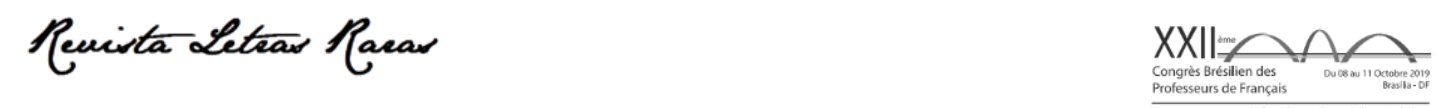

Actes du XXIlème Congrès brésilien des professeurs de français (dans Édition spéciale de la Revue Letras Raras 2020)

ISSN : 2317-2347 - v. 9, Dossier Spécial (2020)

Todo o conteúdo da RLR está licenciado sob Creative Commons Atribuição 4.0 Internacional

Les termes race et ethnie sont devenus des catégories sociales qui viennent s'ajouter au terme genre dans les théorisations sur les inégalités. Ces identités sociales produisent des différentes exclusions, oppressions et violences (HOOKS, 2015). Ferreira (2012, p. 24), comprend la « race comme socialement et historiquement construite » alors que l'ethnie « fait référence à des groupes qui partagent la même identité culturelle, comme la langue, la religion et l'histoire, par exemple ».

Au Brésil, il persiste l'image d'un pays mixte où les différences raciales sont moins évidentes. C'est le mythe de la démocratie raciale toujours présent chez nous. Malgré le fait d'être le deuxième pays noir au monde, derrière le Nigéria (HERINGER, 2001) nous sommes un pays très raciste.

L'anthropologue Telles (2003) souligne qu'au Brésil la race est surtout basée sur la couleur de la peau et dans les aspects physiques. Dans le contexte européen, Santerini (2015) signale la montée du racisme, de la xénophobie et des intolérances, plus particulièrement à l'égard des migrants, des musulmans, des juifs et des Roms. Selon l'auteure, à part quelques différences du racisme traditionnel, basé sur les caractéristiques biologiques, l'Europe vit un néo-racisme, fondé sur les différences culturelles, responsables pour augmenter l'intolérance.

\section{Le contexte de la recherche}

Cette étude est ancrée sur la recherche-action (BARBIER, 2007) qui prévoit des actions de transformation de la réalité et construit des connaissances correspondantes à cette transformation. Elle cherche à développer une pratique critique avec la participation directe du chercheur et des participants dans la recherche. De plus, elle reconnaît les incertitudes, les émotions et les possibilités de réfléchir et refaire nos pratiques.

La recherche s'est développée au 1er semestre de 2016 à l'Alliance Française (AF) de Brasilia grâce au partenariat existant depuis 1966 entre le Secrétariat d'État d'Éducation du District Fédéral (SEEDF) et l'AF de Brasilia. Cet accord prévoit que les enseignants de FLE du réseau public du DF soient aussi affectés à l'AF. En revanche, les apprenants du réseau public peuvent y étudier sans aucun coût. Cet accord permet donc que des apprenants de différentes classes sociales puissent partager le même contexte d'apprentissage du français et interagir entre eux. 

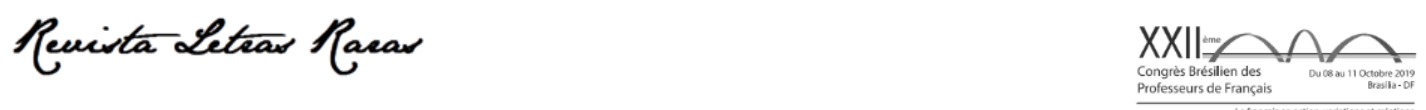

Actes du XXIlème Congrès brésilien des professeurs de français (dans Édition spéciale de la Revue Letras Raras 2020)

ISSN : 2317-2347 - v. 9, Dossier Spécial (2020)

Todo o conteúdo da RLR está licenciado sob Creative Commons Atribuição 4.0 Internacional

De cette façon, moi, en tant qu'enseignante du réseau public du SEEDF j'ai pu y travailler et développer ma recherche de Master Recherche. J'avais un groupe d'adolescents entre 14 ans et 17 ans, 6 filles et 7 garçons en niveau B1 de français, ce qui m'a permis de mieux développer les conversations sur les thèmes abordés dans cette étude. Ci-dessous, il est possible d'avoir plus de détails sur les apprenants (Tab.1). Leur prénom a été modifié par un pseudonyme choisi par eux-mêmes.

Tableau 1 : Profil des participants de la recherche ${ }^{2}$

\begin{tabular}{|c|c|c|c|c|c|}
\hline Nom & Nationalité & Âge & Sexe & Couleur & École \\
\hline Alexandre Agrigento & Portugaise & 15 ans & $\mathrm{M}$ & Blanche & Privée \\
\hline Anna W & Brésilienne & 15 ans & $\mathrm{F}$ & Blanche & Privée \\
\hline Biel & Brésilienne & 15 ans & $\mathrm{M}$ & Brune & Publique \\
\hline Cléo & Brésilienne & 15 ans & $\mathrm{F}$ & Blanche & Privée \\
\hline Johnson & Brésilienne & 16 ans & $\mathrm{M}$ & Brune & Privée \\
\hline José & Brésilienne & 14 ans & $\mathrm{M}$ & Blanche & Privée \\
\hline Lara & Brésilienne & 16 ans & $\mathrm{F}$ & Blanche & Publique \\
\hline Lauren & Brésilienne & 15 ans & $\mathrm{F}$ & Blanche & Privée \\
\hline Lenor Estevão & Portugaise & 16 ans & $\mathrm{F}$ & Blanche & Privée \\
\hline Manoca & Brésilienne & 17 ans & $\mathrm{M}$ & Jaune & Publique \\
\hline Roberta & Brésilienne & 16 ans & $\mathrm{F}$ & Autre & Privée \\
\hline Robervaldo & Brésilienne & 16 ans & $\mathrm{M}$ & Brune & Publique \\
\hline Washington & Brésilienne & 16 ans & $\mathrm{M}$ & Blanche & Privée \\
\hline Source: crepar l'auteure & & & & \\
\hline
\end{tabular}

Source : crée par l'auteure

Nous avons utilisé le Manuel Amis e Compagnie 4 de CLE Internationale. Je cherchais surtout à aller au-delà de ce qui était prévu par le manuel, j'essayais d'attirer l'attention des apprenants sur les images et sur les textes y présents. En plus, j'avais préparé dix activités sous forme de fiche pédagogique sur les thèmes du genre et de la race/ethnie. Les cours ont été enregistrés, et j'avais également un journal où j'écrivais mes impressions à la fin de chaque cours. Après les activités, les apprenants ont répondu à un questionnaire afin de décrire leurs impressions sur le thème proposé et la discussion menée en classe. Ils ont aussi fait des productions écrites sur les thématiques abordées.

\section{Les données de la recherche}

2 Dans le tableau 1, la sigle $\mathbf{M}$ indique masculin et la sigle $\mathbf{F}$ indique féminin.

3 L'apprenant Manoca s'est identifié en tant que jaune pourtant il n'était pas asiatique. 

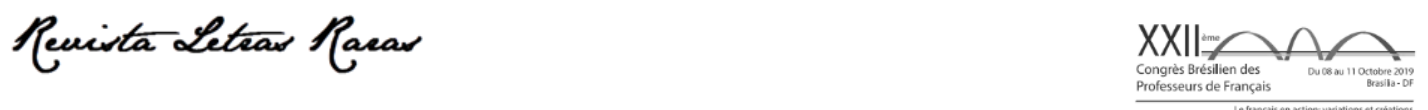

Actes du XXIlème Congrès brésilien des professeurs de français (dans Édition spéciale de la Revue Letras Raras 2020)

ISSN : 2317-2347 - v. 9, Dossier Spécial (2020)

Todo o conteúdo da RLR está licenciado sob Creative Commons Atribuição 4.0 Internacional

Dans ce travail, trois activités ont été analysées. L'activité II sur le féminicide, l'activité V sur la construction des genres féminin et masculin, et l'activité IX sur le racisme en France.

Les apprenants se sont interagis surtout en langue française. Pourtant, des fois, ils se sont exprimés en portugais. Pour la transcription des audios, des symboles ont été utilisés. ${ }^{4}$

\subsection{Activité II : Le féminicide}

Au début du semestre, j'avais montré aux apprenants une photo de l'écrivaine française Simone de Beauvoir avec sa citation : " on ne naît pas femme, on le devient ». La phrase a déclenché des réflexions autour de la construction des rôles de la femme dans les différentes sociétés.

Après quelques réflexions, nous avons travaillé avec le manuel. Dans la leçon, il y avait la musique Madeleine de Jacques Brel écrite dans les années soixante. La chanson a comme personnage principal un homme qui attend tous les jours Madeleine à la même place. II imagine tout ce que les deux pourraient faire ensemble, les lieux à visiter. Pourtant Madeleine ne vient jamais à cette rencontre.

Afin de problématiser l'attitude de ce personnage dans la chanson, j'ai posé des questions aux apprenants, telles que :

\section{Interaction 1}

Professeure : Qu'est-ce que vous pensez de cet homme ? II est normal ?

Élèves : lludé... Drogas.

Washington : II a des problèmes.

Lauren : Madeleine c'est un ami imaginaire.

Roberta : C'est un fatôme.

Professeure : C'est une amie imaginaire?

Élèves : Oui.

Lauren : C'est ça Madeleine.

Professeure : Donc il vit dans l'illusion...

Élèves : Oui.

Professeure : C'est un psychopathe?

Johnson : Sociopathe.

Professeure : Mais le sociopathe c'est différent, non ? Qu'est-ce que c'est un sociopathe?

\footnotetext{
${ }^{4}$ Symboles utilisés dans la trasncription des audios :

... Indiquent un declin dans l'entonation de la phrase suivi d'une pause.

(...) Indiquent une coupure dans la parole.

[] Indiquent un commentaire de la professeure-chercheuse.
} 

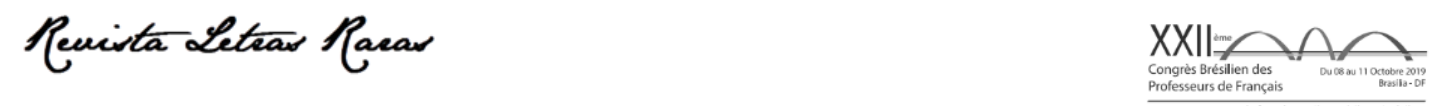

Actes du XXIlème Congrès brésilien des professeurs de français (dans Édition spéciale de la Revue Letras Raras 2020)

ISSN : 2317-2347 - v. 9, Dossier Spécial (2020)

Todo o conteúdo da RLR está licenciado sob Creative Commons Atribuição 4.0 Internacional

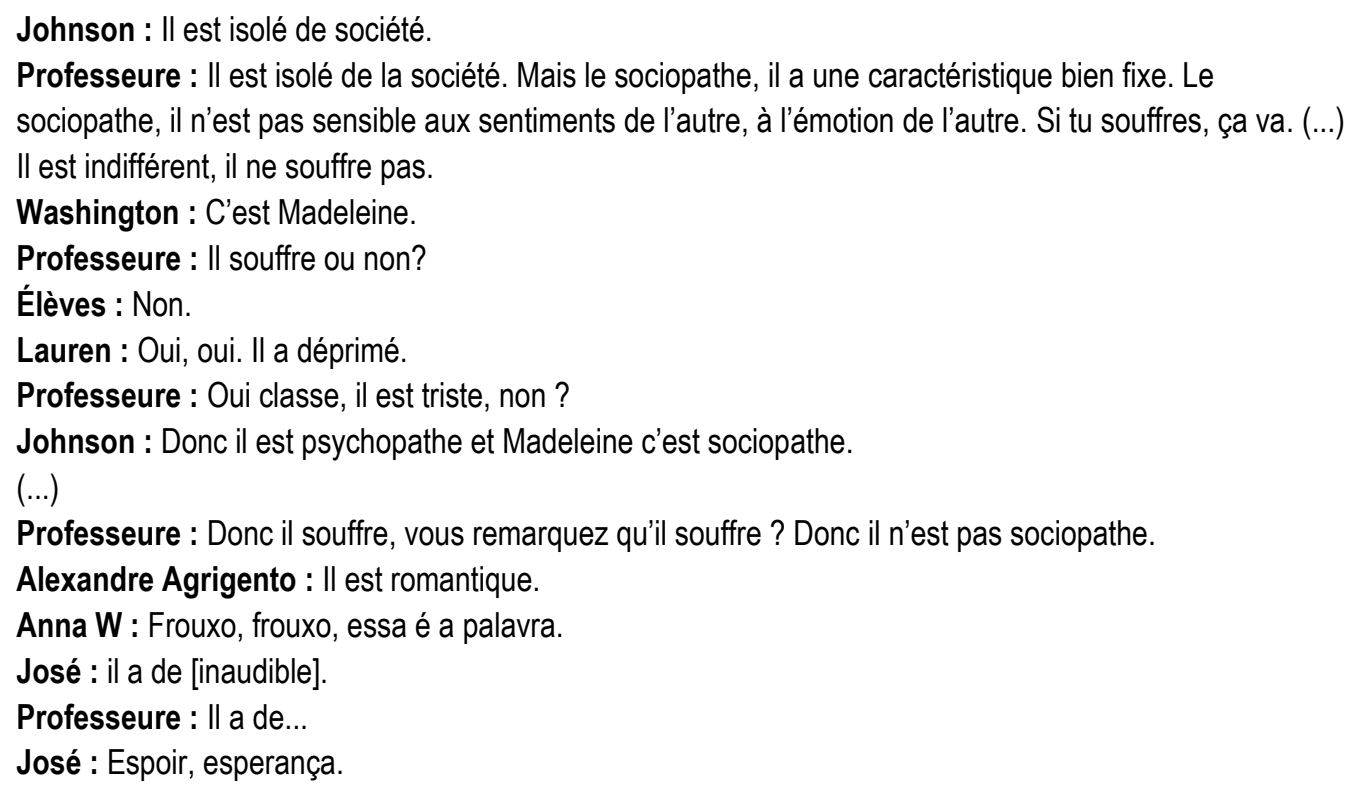

Dans l'interaction 1, au moment où j'ai utilisé l'adjectif « normal », j'ai fait référence à une image figée de l'homme, qui ne prévoit pas qu'il puisse attendre une femme. Inconsciemment, j'ai fait référence à une image socialement construite et stéréotypée de I'homme, ce qui nous montre que ces images font aussi partie de notre inconscient.

II est possible d'observer que les apprenants suivent ma pensée et, sans me poser des questions, ils répondent que l'homme a des illusions. Lauren et Roberta semblent imaginer que Madeleine n'existe même pas puisqu'elle ne vient jamais rencontrer l'homme qui l'aime. Whashington est arrivé à la conclusion que Madeleine était forcément une sociopathe. Or, en aucun moment les apprenants ont réagi pour signaler le pouvoir de décision de Madeleine de ne pas vouloir venir à cette rencontre.

De la même façon, Johnson semble voir les attitudes de l'homme et de la femme comme des conséquences des troubles psychologiques une fois qu'elles ne correspondent pas aux représentations de deux genres dans la société. Son discours va dans le même sens de l'image de ces collègues puisque l'attitude de l'homme sort de la norme sociale prévue pour lui. Cette vision est aussi confirmée par Anna W.

Cependant, il n'y a eu que deux apprenants, Alexandre Agrigento et José, qui ont apporté un point de vue différent de celui de la norme sociale. Ils se sont basés ici dans d'autres représentations moins stéréotypées du genre masculin.

Dans cette interaction, les apprenants, dans leur grande majorité, se sont appuyés sur des images figées des genres. Quand les attitudes des personnages ne correspondaient pas à 

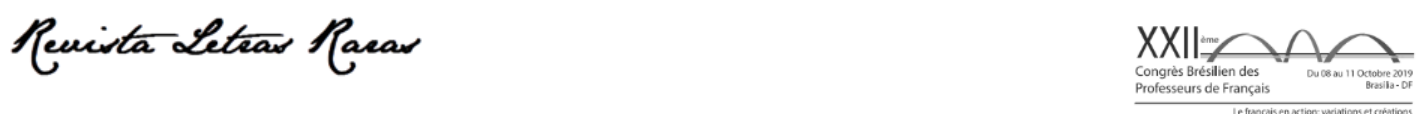

Actes du XXIlème Congrès brésilien des professeurs de français (dans Édition spéciale de la Revue Letras Raras 2020)

ISSN : 2317-2347 - v. 9, Dossier Spécial (2020)

Todo o conteúdo da RLR está licenciado sob Creative Commons Atribuição 4.0 Internacional

ce qui était prévue, ils les ont interprétés comme des conséquences d'une maladie. Ce type de pensée est d'ailleurs très répandue dans notre société. En général, les gens n'arrivent pas à identifier les effets négatifs du machisme, surtout en ce qui concerne l'apprentissage des rôles féminins et masculins. II y a des expectatives sur ce que hommes et femmes peuvent ou doivent faire et sur comment le faire. On a une tendance à négliger les conséquences de ces constructions dans notre vie.

Dans le dialogue suivant, on peut observer tout cela lors d'une discussion sur un cas de féminicide au Brésil :

\section{Interaction 2}

Professeure : Classe, qu'est-ce qui mène un homme à rompre, à tuer une femme seulement parce qu'elle ne veut pas être avec lui ?

Lauren : É... Como é que fala fica?

Professeure : Fica em que sentido?

Lauren : Fica agressivo.

Professeure : Il devient agressif.

Lauren : Il devient agressif.

Professeure : Mais pourquoi il n'accepte pas?

Anna W : Il est descontrolé, il est crazy.

Élèves : [ils rient]

Professeure : Il est fou.

Anna W : Como se dit louco?

Professeure : Fou.

Anna W : Fou, fou.

Professeure : Mais donc vous pensez que tous les hommes qui tuent les femmes, c'est parce qu'ils sont fous?

Roberta : Covarde.

Professeure : lls sont lâches [en écrivant au tableau]. (...) Donc, ce n'est pas simplement parce qu'ils sont fous, c'est parce qu'ils sont lâches. Et pourquoi ils n'acceptent pas un « non »? Quel est le problème?

Luren : Parce que, ham, quer...

Professeure : Il veut...

Lauren : Il veut être... Como é que é?

Roberta : Ele se acha superior.

Professeure : Ah, ils se sentent supérieurs [en écrivant au tableau] à la femme, oui ?

Lauren : Oui.

Professeure : Vous êtes d'accord ? Alexandre Agrigento, tu voudrais dire quelque chose?

Alexandre Agrigento : Non, non, non.

Professeure : Et pourquoi ils se sentent supérieurs?

Johnson : Parce qu'il y a un sentiment de posse.

José : Machisme. 

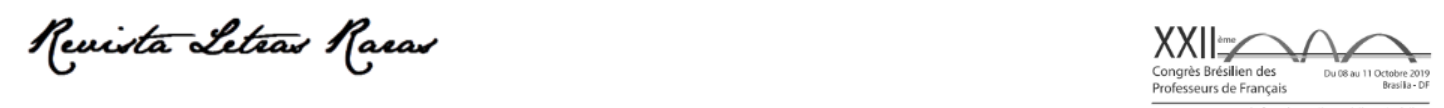

Actes du XXIlème Congrès brésilien des professeurs de français (dans Édition spéciale de la Revue Letras Raras 2020) ISSN : 2317-2347 - v. 9, Dossier Spécial (2020)

Todo o conteúdo da RLR está licenciado sob Creative Commons Atribuição 4.0 Internacional

Dans cette interaction, mon objectif est de problématiser les discours des apprenants. En leur demandant à chaque fois « pourquoi », j'essayais de les pousser à réfléchir encore une fois sur ce qu'ils venaient de dire, ou alors sur le discours d'un collègue, ce qui les menaient à réfléchir ensemble. Dans l'interaction 2, les apprenants sont arrivés à voir que les violences contre les femmes ne sont pas nécessairement dues à des troubles psychologiques, mais plutôt à des constructions sociales et notamment au machisme qui empêche les femmes, et aussi les hommes, d'être libres dans leur façon d'être.

II est possible de voir que le manuel a favorisé le travail dans la perspective de la LC tout en problématisant les identités y présentes, comme propose Duboc (2015). Pour cela, il est essentiel de former les enseignants à l'éducation interculturelle vers la citoyenneté.

\subsection{Activité V : C'est quoi le genre?}

Afin de mieux comprendre le thème, nous avons travaillé sur la vidéo : C'est quoi le genre $?^{5}$ dans laquelle il y a quelques exemples sur la construction des genres dans la société à partir des cadeaux d'anniversaire offerts aux filles et aux garçons. Nous avons également discuté sur les adjectifs utilisés tout au long de notre vie. Ils ont la fonction de nous caractériser et aussi de construire nos identités.

Dans la vidéo, il est évident la différence entre les cadeaux offerts aux garçons : un ballon, une fusée et un monstre. Et pour les filles : un poupon, un livre et un aspirateur. Tout cela contribue pour renforcer, depuis l'enfance, les espaces occupés par chacun dans leur vie personnelle et professionnelle. De même, les adjectifs attribuent des qualités et des comportements différents. Voici le dialogue issu de cette interaction :

\section{Interaction 3}

Professeure : Qu'est-ce que ça veut dire ces cadeaux?

Washington : C'est la pression de la société.

Professeure : Quelle est l'importance de ces cadeaux dans la formation du genre ? Dans la manière dont cette personne va se voir?

Lauren : Parce que, hã...

Lara : Les cadeaux construit la personnalité de l'enfant. II va crescer, il va...

Professeure : Quand il va grandir...

Lara : Sa personnalité c'est un reflèxe des cadeaux.

(...)

5 Disponible sur : [https://www.youtube.com/watch?v=xtbDynD7DE8\&t=75s]. Accès : le 25 oct. 2020. 

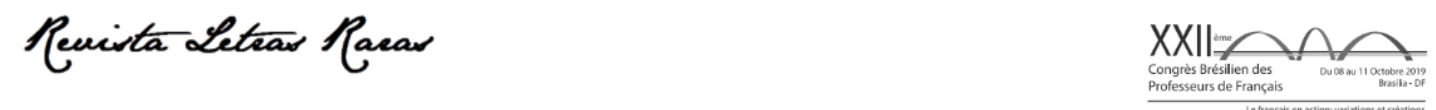

Actes du XXIlème Congrès brésilien des professeurs de français (dans Édition spéciale de la Revue Letras Raras 2020)

ISSN : 2317-2347 - v. 9, Dossier Spécial (2020)

Todo o conteúdo da RLR está licenciado sob Creative Commons Atribuição 4.0 Internacional

Professeure : Par exemple, on donne la poupée, vous comprenez une poupée?

Élèves : Oui.

Professeure : On donne une poupée à une fille, c'est très commun de donner une poupée à une fille.

Élèves : Oui.

Professeure : Pourquoi?

Manoca : Parce qu'elle va ser mère.

Professeure : Et on peut donner une poupée à un garçon?

Élèves : Oui.

Professeure : Est-ce que vous donneriez une poupée à un garçon ? (...)

José : Non.

Roberta : Un X Men.

Élèves : Un Max Steel.

Professeure : C'est commun de donner une poupée à un garçon?

Élèves : Non.

Professeure : Pourquoi non?

Johnson : Poupée ? Qu'est-ce que c'est poupée?

Élèves : Uma boneca.

Washington : Parce que c'est l'influence...

II est intéressant de remarquer que Lara semble comprendre l'influence des ces cadeaux dans la construction des identités de l'enfant et plus tard dans sa vie adulte. Les apprenants montrent également comprendre la culture au moment d'offrir un cadeau à un enfant. Ils sont tous d'accord pour dire qu'il est possible d'offrir une poupée à un garçon mais face à ma question s'ils donneraient une poupée à un garçon, José se précipite pour dire « non ".

Dans la vidéo visionnée au préalable, il est possible d'observer que le genre permet de pointer les différences qui ne sont ni biologiques ni innées, mais socialement construites. Le genre est donc un important outil d'analyse pour comprendre comment se construisent les inégalités entre les femmes et les hommes. Le genre est construit dans et par la langue car les identités sont construites dans le discours par le langage et les représentations faites à partir des systèmes symboliques (WOODWARD, 2014). II a été possible de conclure que les façons de se porter et d'agir dans la société est résultat d'un apprentissage fait à partir du sexe de l'enfant.

\subsection{Activité IX : Le racisme ordinaire}



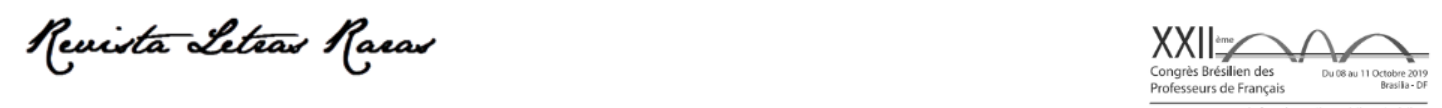

Actes du XXIlème Congrès brésilien des professeurs de français (dans Édition spéciale de la Revue Letras Raras 2020)

ISSN : 2317-2347 - v. 9, Dossier Spécial (2020)

Todo o conteúdo da RLR está licenciado sob Creative Commons Atribuição 4.0 Internacional

Cette activité a été développée dans l'objectif de réfléchir sur le racisme en France. Nous avons déclenché la conversation à partir d'une vidéo nommée Les mots qui font mal6. La vidéo a été réalisée en France afin de dénoncer le racisme ordinaire, celui qui s'exprime tous les jours. Ce racisme est renforcé par l'humour, par une petite phrase ou même une question anodine de manière consciente ou inconsciente. II est bien plus difficile à identifier et à dénoncer qu'une agression physique. Néanmoins, il constitue à petites doses répétées une violence quotidienne pour beaucoup de Français.

Dans la vidéo, il y a des gens de plusieurs nationalités, couleurs et religions qui sont constamment discriminés par leurs différences culturelles. Des phrases telles que : «Tu parles français comme un blanc »; "Tu parles africain ? "; "Tu dis que t'es français mais tu viens d'où ? ", entre autres, sont répétées dans le but de montrer de quelle manière le racisme ordinaire est présent dans les pratiques culturelles et surtout dans la langue française.

Dans cette activité, il a été possible de faire un parallèle entre le racisme brésilien, lié surtout aux caractéristiques phénotypiques (TELLES, 2003), et celui trouvé en Europe, où le « racisme culturel » a lieu non seulement à cause de la couleur mais aussi des différences culturelles (SANTERINI, 2015, p. 7).

Avant de faire regarder la vidéo, j'ai invité les apprenants à réfléchir sur le concept de racisme pour avoir leurs perceptions du racisme en France :

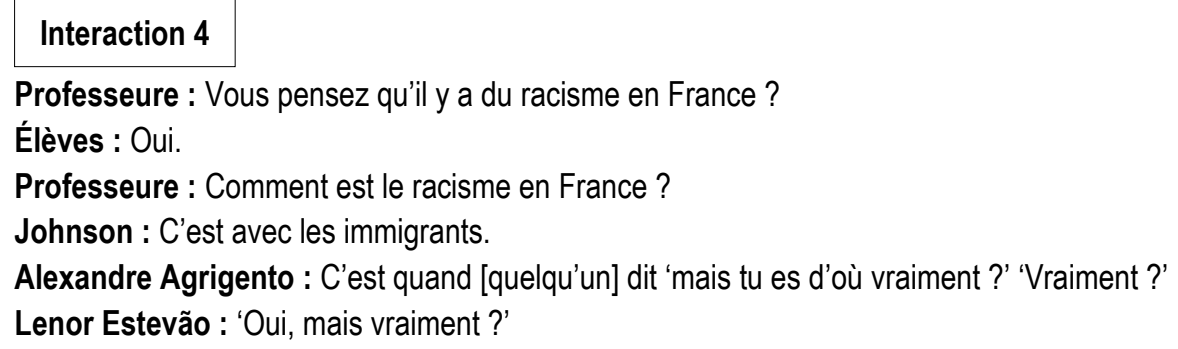

L'apprenant Johnson présente une compréhension du racisme apporté par Santerini (2015). De même, Alexandre Agrigento et Lenor Estevão évoquent les mots qui font mal et qui sont constamment répétés envers les Français d'origine africaine, par exemple. Ensuite, nous avons regardé la vidéo. Voici l'interaction faite après le visionnage :

\footnotetext{
6 Disponible sur : [https://www.youtube.com/watch?v=QfpuFyf3nRk]. Accès : le 20 oct. 2020.
} 

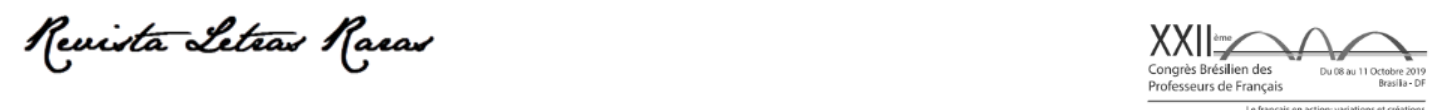

Actes du XXIlème Congrès brésilien des professeurs de français (dans Édition spéciale de la Revue Letras Raras 2020)

ISSN : 2317-2347 - v. 9, Dossier Spécial (2020)

Todo o conteúdo da RLR está licenciado sob Creative Commons Atribuição 4.0 Internacional

\section{Interaction 5}

Professeure : De quoi il s'agit cette vidéo ? Qu'est-ce que vous avez vu dans cette vidéo?

Johnson : Des phrases, des préjugés...

Élèves : Des stéréotypes.

José : Beaucoup de choses mauvais pour une personne.

Professeure : Qui font mal, oui?

Cléo : Il y a aussi la marginalisation des personnes nées en France mais filles d'immigrants. Et la société française... faz você se sentir?

Professeure : La société française les fait sentir...

Cléo : Les fait sentir qu'lls ne sont pas français.

Professeure : Est-ce qu'il y a du racisme ici [dans la vidéo]?

Élèves : Oui.

Professeure : Et comment le racisme, il est exprimé en France, d'après la vidéo ?

Manoca : lls comparent é... Eles...

Lara : Les questions.

Manoca : Les pays.

Professeure : Parce que le racisme au Brésil, il est très lié à la... ?

Johnson : Le visage.

Professeure : La couleur et aux phénotypes (...) En France, le racisme, il est lié à quoi ?

Manoca : La nationalité.

Roberta : À descendência.

II est clair la valorisation de la culture française dans la vidéo. Après l'avoir regardée, les apprenants montrent comprendre la manière dont le racisme est présent en France. Leurs perceptions renforcent le "néo-racisme "(SANTERINI, 2015, p. 1) trouvé en Europe. Différemment du racisme traditionnel basé sur la hiérarchie des races, ce nouveau racisme est aussi appelé par l'auteure de « racisme sans race » (SANTERINI, 2015, p. 6). II est basé sur une hiérarchie de cultures puisque le concept de race a été nié par la science. Ce racisme est plus insidieux par rapport au racisme traditionnel, pourtant il a les mêmes effets : « il vise à expliquer et légitimer des comportements ou discours discriminatoires, et contribue à les alimenter » (SANTERINI, 2015, p. 6).

Le racisme est lié à plusieurs facteurs. La vidéo nous a permis de repenser les différentes manières par lesquelles il peut se trouver en France, ainsi que l'impact de ces discours envers des groupes de personnes.

\section{En guise de conclusion}

Dans cette étude, les apprenants ont réfléchi sur des thèmes sociaux dans le cours de FLE. En ce qui concerne leurs perceptions de la réalité, il y a eu des moments où ils ont 

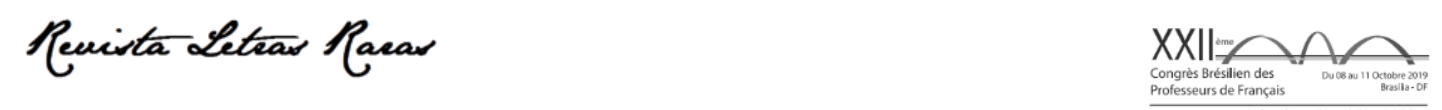

Actes du XXIlème Congrès brésilien des professeurs de français (dans Édition spéciale de la Revue Letras Raras 2020) ISSN : 2317-2347 - v. 9, Dossier Spécial (2020)

Todo o conteúdo da RLR está licenciado sob Creative Commons Atribuição 4.0 Internacional

apporté des représentations basées sur le discours commun de la société, ce qui nous a permis de réfléchir ensemble sur les différentes formes de discrimination. Ils ont aussi fait preuve d'un regard critique envers les groupes défavorisés. Les apprenants des écoles privées ont interagi beaucoup plus et ont montré une vision plus complexe envers les situations de la vie quotidienne. À mon avis, cela se doit aux différents contextes d'apprentissage où ils se trouvent, soit à l'école, soit à la maison. Au Brésil, le manque de capital économique, social et culturel (BOURDIEU, 1994) chez les apprenants du réseau publique influencent leur manière d'agir dans le monde ainsi que leur perception de la réalité.

La littératie critique, ainsi que l'enseignement interculturel, nous a permis de réfléchir sur des situations de la vie. Nous avons travaillé le manuel pendant tout le semestre et avons profité de cet outil d'apprentissage pour déclencher des conversations autour des thèmes. Pour cela, il est primordial que l'enseignant soit attentif aux plusieurs discours afin de favoriser la problématisation. D'ailleurs, la façon d'agir en classe a été un grand défi, je me demandais souvent comment réagir face aux discours des apprenants, quelles questions leur poser et comment mener la conversation. À la fin de l'étude, j'ai compris qu'il n'y a pas une seule façon d'agir en classe, et que l'enseignant ne devrait pas chercher à convaincre ses élèves de quoi que ce soit mais de les faire penser à la construction de leurs identités à partir des aspects politiques, historiques et culturels présents dans ce processus.

Ce qui est intéressant de remarquer, c'est que les apprenants se sont servis de la langue française non seulement pour développer leurs connaissances linguistiques mais aussi les aspects culturels qui sont attachés à la langue. Ils étaient confidents et à l'aise pour participer aux interactions. Cela dit, cette pratique a favorisé l'apprentissage de la langueculture et les ont mis dans le rôle d'acteurs sociaux qui pensent leur entourage et agissent dans le monde.

Tout cela nous amène aux réflexions de Santerini $(2015$, p. 1) où elle insiste qu' « il convient de combattre l'intolérance en utilisant des outils culturels et sociaux. L'éducation et l'information doivent jouer un rôle crucial dans la formation des citoyens au respect de la diversité" ». C'est justement ce type d'éducation libre (FREIRE, 2011) et solidaire que nous aimerions faire revivre dans nos cours de français.

\section{Références}



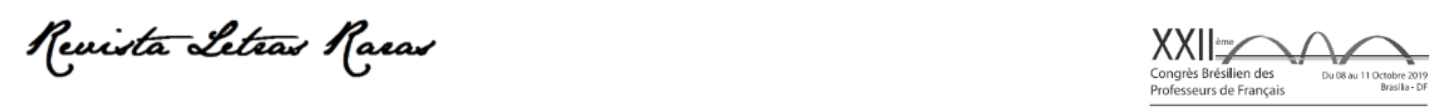

Actes du XXIlème Congrès brésilien des professeurs de français (dans Édition spéciale de la Revue Letras Raras 2020) ISSN : 2317-2347 - v. 9, Dossier Spécial (2020)

Todo o conteúdo da RLR está licenciado sob Creative Commons Atribuição 4.0 Internacional

BARBIER, R. A Pesquisa-Ação. Tradução Lucie Didio. Brasília: Liber Livro Editora, 2007.

BEAUVOIR, S. O Segundo Sexo. 2. ed. Rio de Janeiro: Nova Fronteira, 2009.

BOURDIEU, P. A Economia das Trocas Lingüísticas. In: ORTIZ, R. Pierre Bourdieu: sociologia. São Paulo: Ática, 1994.

BYRAM, M ; GRIBKOVA, B ; STARKEY, H. Développer la dimension interculturelle dans l'enseignement des langues : une introduction pratique à l'usage des enseignants. Strasbourg : Conseil de l'Europe, 2002. Disponível em: [https://rm.coe.int/16802fc3aa]. Acesso em: 14 out. 2020.

DUBOC. A. P. Letramento crítico nas brechas da sala de línguas estrangeiras. In: TAKAKI, N. H.; MACIEL, R. F. (Orgs.). Letramentos em terra de Paulo Freire. 2. ed. São Paulo: Pontes Editores, 2015. p. 209-229.

FAIRCLOUGH, N. Discurso e mudança social. Tradução de Izabel Magalhães. Brasília: Editora Universidade de Brasília, 2008.

FERREIRA, A. J. Identidades sociais de raça/etnia na sala de aula de língua inglesa. In: Identidades sociais de raça, etnia, gênero e sexualidade: práticas pedagógicas em sala de aula de línguas e formação de professores/as. São Paulo: Pontes Editores, 2012. p. 1950.

FREIRE, P. Pedagogia do oprimido. 50. ed. Rio de Janeiro: Paz e Terra, 2011.

HALL, S. A identidade cultural na pós-modernidade. Tradução Tomaz Tadeu da Silva. Rio de Janeiro: Lamparina, 2014.

HERINGER, R. Mapeamentos das ações e discursos de combate às desigualdades raciais no Brasil. Estudos Afro-Asiáticos, Rio de Janeiro, v. 23, n. 2, p. 1-43, 2001.

HOOKS, B. Ensinando a transgredir: a educação como prática da liberdade. São Paulo: WMF Martins Fontes, 2013.

Mulheres negras: moldando a teoria feminista. Tradução de Roberto Cataldo Costa.

Revista Brasileira de Ciência Política, Brasília, n. 16, p. 193-210, jan./ abr. 2015.

MENEZES DE SOUSA, L. M. T. Para uma redefinição de letramento crítico: conflito e produção de significação. In: MACIEL, R. F.; ARAÚJO, V. A. (Org.) Formação de professores de línguas: ampliando perspectivas. Jundiaí: Paco Editorial, 2011.

MOITA LOPES, L. P. Linguagem e escola na construção de quem somos. In: FERREIRA, A. J. (Org.). Identidades sociais de raça, etnia, gênero e sexualidade: práticas pedagógicas em sala de aula de línguas e formação de professores/as. São Paulo: Pontes Editores, 2012. p. 9-12.

MONTE MÓR, W. Crítica e letramentos críticos: reflexões preliminares. In: ROCHA, C. H.; MACIEL, R. F. (Orgs.). Língua estrangeira e formação cidadã: por entre discursos e práticas. São Paulo: Pontes Editores, 2015. p. 31-50.

NORTON, B. Identity and Language Learning: Gender, Ethnicity and Educational Change. Harlow, England: Pearson Education, 2000.

PENNYCOOK, A. Critical Applied Linguistics: a critical introduction. Mahwah, NJ: Lawrence Erlbaum Associates, 2001.

PUREN, C. Perspective actionnelle et plurilinguisme: pour un traitement didactique et non sociolinguistique du plurilinguisme scolaire au Maghreb. Disponível em: [https://www.christianpuren.com/mes-travaux/2018g/]. Acesso em: 10 out. 2020. 

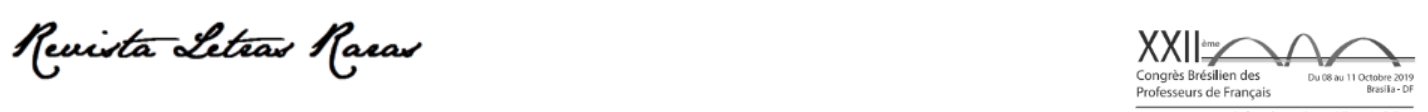

Actes du XXIlème Congrès brésilien des professeurs de français (dans Édition spéciale de la Revue Letras Raras 2020)

ISSN : 2317-2347 - v. 9, Dossier Spécial (2020)

Todo o conteúdo da RLR está licenciado sob Creative Commons Atribuição 4.0 Internacional

SANTERINI, M. Reconnaître et prévenir le néo-racisme. Strasbourg: Conseil de l'Europe, 2015. Disponivel em: [http://assembly.coe.int/nw/xml/XRef/Xref-DocDetails-fr.asp?Fileld=21803]. Accesso em: 15 out. 2020.

SCOTT, J. Gênero: uma categoria últil para análise histórica. Educação e Realidade, Porto Alegre, v. 20, n. 2, p. 71-79, jul./dez. 1995.

SILVA, T. T. (Org.). A produção social da identidade e da diferença. In: Identidade e diferença: a perspectiva dos estudos culturais. 15. ed. Petrópolis: Vozes, 2014. p. 73-102.

SOARES, M. Letramento: um tema em três gêneros. Belo Horizonte: Autêntica, 1998.

TELLES, E. Racismo à brasileira: uma nova perspectiva sociológica. Rio de Janeiro: Relume Dumará, 2003.

THOMPSON, J. B. Ideologia e cultura moderna: teoria social crítica na era dos meios de comunicação de massa. 2. ed. Petrópolis: Vozes, 1990.

WOODWARD, K. Identidade e diferença: uma introdução teórica e conceitual. In: SILVA, T. T. (Org.). Identidade e diferença: a perspectiva dos estudos culturais. 15. ed. Petrópolis: Vozes, 2014. p. 07-72. 